\title{
Quantitative-Profiling of Neurotransmitter Abnormalities in Disease Progression of Experimental Diabetic Encephalopathy Rat
}

\begin{tabular}{|r|l|}
\hline Journal: & Canadian Journal of Physiology and Pharmacology \\
\hline Manuscript ID: & cjpp-2015-0118.R1 \\
\hline Manuscript Type: & Article \\
\hline Date Submitted by the Author: & $16-$ May-2015 \\
\hline Complete List of Authors: & $\begin{array}{l}\text { Zhou, Xueyan; Xuzhou Medical College, Key Laboratory of New Drug } \\
\text { Research and Clinical Pharmacy } \\
\text { Zhu, Qiuxiang; Xuzhou Medical College, Key Laboratory of New Drug } \\
\text { Research and Clinical Pharmacy } \\
\text { Han, Xiaowen; Xuzhou Medical College, Key Laboratory of New Drug } \\
\text { Research and Clinical Pharmacy } \\
\text { Chen, Renguo; Xuzhou Medical College, Key Laboratory of New Drug } \\
\text { Research and Clinical Pharmacy } \\
\text { Liu, Yao-Wu; Xuzhou Medical College, Key Laboratory of New Drugs and } \\
\text { Clinical Application } \\
\text { Fan, Hongbin; Affiliated Hospital of Xuzhou Medical College, Department of } \\
\text { Neurology } \\
\text { Yin, Xiao-Xing; Xuzhou Medical College, Key Laboratory of New Drugs and } \\
\text { Clinical Application }\end{array}$ \\
\hline Keyword: & $\begin{array}{l}\text { diabetic encephalopathy (DE), cognitive dysfunction, acetylcholine (ACh), } \\
\text { glutamate (Glu), Y-aminobutyric acid (GABA) }\end{array}$ \\
\hline
\end{tabular}




\title{
Quantitative-Profiling of Neurotransmitter Abnormalities in Disease Progression of Experimental Diabetic Encephalopathy Rat
}

\author{
Xueyan Zhou ${ }^{a^{*}}$, Qiuxiang Zhu ${ }^{\mathrm{a}^{*}}$, Xiaowen Han ${ }^{\mathrm{a}}$, Renguo Chen ${ }^{\mathrm{a}}$, Yaowu Liu ${ }^{\mathrm{a}}$, \\ Hongbin Fan ${ }^{\mathrm{b}}$, Xiaoxing Yin ${ }^{\mathrm{a}}$ \\ ${ }^{a}$ Jiangsu Key Laboratory of New Drug Research and Clinical Pharmacy, Jiangsu \\ Center for the Collaboration and Innovation of Cancer Biotherapy, Xuzhou Medical \\ College, Tongshan Road 209\#, 221004, Xuzhou, China. \\ ${ }^{b}$ Department of Neurology, Affiliated Hospital of Xuzhou Medical College, \\ Huaihai West Road 99\#, 221004, Xuzhou, China. \\ *These authors contributed equally to this work.
}

\section{Corresponding Author:}

Prof. Xiaoxing Yin, Jiangsu Key Laboratory of New Drug Research and Clinical Pharmacy, Jiangsu Center for the Collaboration and Innovation of Cancer Biotherapy, Xuzhou Medical College, 209 Tongshan Road, Xuzhou 221004, China. E-mail:yinxx@xzmc.edu.cn_ Phone: +86 51683262630

Fax: +8651683262630 


\section{Abstract}

Diabetic encephalopathy (DE) is one of the most prevalent chronic complications of diabetes mellitus (DM) with neither effective prevention nor proven therapeutic regimen. The present study aims to uncover the potential dysregulation pattern of the neurotransmitters in the streptozotocin (STZ)-induced experimental DE rat. For that purpose, male Sprague-Dawley (SD) rats were treated with a single intraperitoneal injection of STZ. Cognitive performance was detected by morris water maze (MWM) tests. Serum, cerebrospinal fluid (CSF) and brain tissues were collected for neurotransmitter determination. Compared with control rats, the acetylcholine (ACh) levels in serum, CSF, hippocampus and cortex were all down-regulated significantly as early as 6 weeks in STZ group. In contrast, the glutamate (Glu) levels were decreased in CSF and hippocampus, but unaffected in serum and cortex of STZ rats. As for $\gamma$-aminobutyric acid (GABA), it was down-regulated in serum, but up-regulated in CSF, hippocampus and cortex in STZ group. The mRNA expressions of neurotransmitter related rate limiting enzymes (including AChE, GAD1 and GAD2) and pro-inflammatory cytokines (including IL-1 $\beta$ and TNF- $\alpha$ ), were all increased in DE rats. Our data suggests that DM induces an isoform-dependent and tissue-specific neurotransmitter abnormalities, and the neuroinflammation may underlay the nervous system dysfuction observed in DE progression.

Keywords: cognitive dysfunction, diabetic encephalopathy (DE), neurotransmitter, acetylcholine (ACh), glutamate (Glu), $\gamma$-aminobutyric acid (GABA), neuroinflammation, acetylcholinesterase (AChE), glutamate decarboxylase (GAD) 


\section{Introduction}

Diabetes mellitus (DM) is one of the most devastating chronic systemic diseases. DM can damage several organs of the body, a less addressed and not well-recognized complication is diabetic encephalopathy(DE) (McCrimmon 2012), with cognitive dysfunction as its core component(Biessels et al. 2008). Hyperglycemia, vascular disease, and altered insulin signaling may play a significant role in the pathophysiology of cognitive impairment, but the mechanism of DE looks complex and yet not completely understood(Kodl and Seaquist 2008).

The current consensus is that the preventive actions and therapies for DE should be started as soon as possible in order to be effective. This emphasizes the importance of early diagnosis. Until now, most of the cognitive disorders are clinically diagnosed by taking physical and neurological examinations, and checking other signs of intellectual impairment through standard neuropsychological and cognitive tests(Antila et al. 2013). However, diagnosis is known to progress for several years or even decades prior to appearance of the first clear symptoms(Singhal and Sandhir 2015). Thus, early diagnosis of DE remains a great challenge in its long period of progression.

Evidence have shown that neurotransmitter system is implicated in the early pathophysiology of cognitive dysfunction(Prakash et al. 2015), and several neurotransmitter systems have been used as diagnostic indicators of degenerative encephalopathy (Levenga et al. 2013). Cholinergic system is an important modulatory neurotransmitter system that is involved in conscious awareness(Perry et al. 1999). 
One study has indicated that the synthesis and release of acetylcholine (ACh) was significantly decreased when associated with uncontrolled hyperglycemia (Welsh and Wecker 1991). It has also been reported that cholinergic system dysfunction has direct connection with the alteration of crucial brain enzyme activities, such as acetylcholinesterase (AChE), and might be one of the cause of cognitive deficit in in STZ-induced DE rats(Liapi et al. 2010). Glutamatergic system is essential for synaptic communication(Frigerio et al. 2008). Recent evidence has demonstrated the involvement of glutamate (Glu) in the pathogenesis of several neurodegenerative disorders(Alberdi et al. 2010). Reisi et al. found that the extracellular basal level of Glu was down regulated at dentate gyrus of STZ induced diabetic rats(Reisi et al. 2009). In addition, impairments of cognition and synaptic plasticity of diabetic rats might have association with glutamatergic neurotransmission abnormality(Gispen and Biessels 2000). It has been reported that GABAergic system is the major inhibitory neurotransmitter in the brain (Wan et al. 2015). The dysfunction of GABAergic can lead to cognitive deficits in STZ-induced rat model (Ramamoorthi and Lin 2011). Studies also indicated that GABA levels were significantly increased in hippocampus of diabetic rats at 8 weeks after STZ treatment (Jangra et al. 2013).

Neurotransmitter imbalances are closely related with the progression of cognitive dysfunction, but there is less research focus on the relationship between neurotransmitter profiles and the DE progression. Therefore, the present study was designed to uncover the dysregulation pattern of neurotransmitters and their related rate limiting enzymes, in the progression of DE by using the STZ-induced rat model, 
and to find out the possible underlying mechanism of neurotransmitter system dysfunction in DE.

\section{Materials and methods}

\section{Chemicals}

Streptozotocin (STZ), acetylcholine (ACh), glutamate (Glu) and $\gamma$-aminobutyric acid (GABA) were all purchased form Sigma (St. Louis, MO, USA). Water used in the experiment was deionized and purified by a Milli-Q system (Millipore, Bedford, MA, USA). All other chemicals and solvents were of analytical grade or the highest grade commercially available.

\section{Animals}

Male Sprague-Dawley (SD) rats (10 weeks of age) were obtained from Shanghai SLRC Laboratory Animal Co., Ltd (Shanghai, China). The animals were maintained in a temperature and humidity controlled environment with a 12:12 h light/dark cycle. Feed and water were available ad libitum. All animal experimental and surgical procedures were performed in strict accordance with the Guide for the Care and Use of Laboratory Animals. The protocol was approved by the Committee on the Ethics of Animal Experiments of Xuzhou Medical College. All surgery was performed under sodium pentobarbital anesthesia, and all efforts were made to minimize suffering.

\section{Experimental Design}

Rats were randomly divided into two sets: the control group and the STZ group.

DE was induced with a single intraperitoneal injection of STZ $(60 \mathrm{mg} / \mathrm{kg}$, dissolved in 0.1 M citrate buffer at $\mathrm{pH} 4.5$ ). Age-matched control rats received vehicle only. The 
development of diabetes was confirmed by fasting blood glucose (FBG) level using a reagent kit (Jiancheng Bioengineering Institute, Nanjing, China). Rats with FBG levels higher than $11.1 \mathrm{mmol} / \mathrm{L}$ were considered to be diabetic after $72 \mathrm{~h}$ of STZ injection(Liu et al. 2013). After the STZ treatment, cerebrospinal fluid (CSF), serum, hippocampus and cortex were collected at designated times (2, 4, 6, 8 and 10 weeks) for neurotransmitter determination. In details, CSF samples were withdrawn using the cisternal puncture technique. Blood samples were collected via abdominal aorta and then centrifuge at $3000 \mathrm{rpm}$ for 15 minutes at $4^{\circ} \mathrm{C}$ to obtain the serum. The whole brain tissues were removed from the skull, and then the cortex and hippocampus were isolated on ice. All samples were stored at $-80^{\circ} \mathrm{C}$ until assay.

\section{Morris Water Maze Test (MWM test)}

The MWM test was employed for testing learning and memory according to our previous reports (Liu et al. 2012). The test system was afforded by COULOURN INSTRUMENTS (7462 Penn Drive, Allentown PA, 18106). The device consisted of a circular water tank $(150 \mathrm{~cm}$ in diameter and $50 \mathrm{~cm}$ high) filled with water maintained at approximately $24^{\circ} \mathrm{C}$, which was divided into four equal quadrants , and a escape platform $(17 \mathrm{~cm}$ in diameter and $31 \mathrm{~cm}$ high) in one of the quadrants, hidden beneath the surface of the water. The tank was located in a large room where there were several brightly colored cues external to the maze. The cues were visible to the swimming rats and used for spatial orientation. The water maze task was carried out for 5 consecutive days.

\section{Learning test}


A place navigation test was performed wherein the extent of learning was assessed. The rats received four consecutive daily training trials. The rats were trained to find the hidden platform, which remained in a fixed location throughout the test. The trials lasted for a maximum $90 \mathrm{~s}$, and the time to reach the platform (latency time) was recorded.

Memory test

A spatial probe test was performed wherein the extent of memory was assessed. The time spent in the target quadrant indicates the degree of memory that has taken place after learning training. In the probe trail, the hidden platform was removed from the pool, and the rat was placed into the pool from the start location at the quadrant opposite to the former platform. The percentage of time spent in the former platform quadrant and the number of platform crossings were recorded for $90 \mathrm{~s}$ to measure the spatial memory retention.

\section{Neurotransmitter Determination by LC-MS/MS}

Sample preparation and LC-MS/MS analysis were based on the method of Zheng X et al. (Zheng et al. 2012) with some modifications.

\section{Sample preparation}

$100 \mu \mathrm{L}$ of saline solution (containing $50 \mathrm{nmol} / \mathrm{L} \beta$-ACh and $10 \mu \mathrm{mol} / \mathrm{L}$ caffeic acid as internal standard chemicals) were added to $50 \mathrm{mg}$ brain tissue, and then homogenized in an ice bath. $200 \mu \mathrm{L}$ of ice-cold acetonitrile was added to the homogenate, and the mixture was vortexed for 2 min followed by centrifugation at $4^{\circ} \mathrm{C}$ for $10 \mathrm{~min}$ at 14,000 g. $100 \mu \mathrm{L}$ of the supernatant was transferred for 
derivatization. Briefly, $50 \mu \mathrm{L}$ of borate buffer (sodium tetraborate, $100 \mathrm{mM}$ in water) and $50 \mu \mathrm{L}$ of benzoyl chloride $(2.0 \%$ in acetonitrile, v/v) were added to the residue and vortexed under room temperature for $5 \mathrm{~min}$. After centrifugation at 14,000 rpm for $10 \mathrm{~min}$, the supernatant was transferred to the vial and $10 \mu \mathrm{L}$ was injected for analysis. CSF and serum samples $(100 \mu \mathrm{L})$ were spiked with $10 \mu \mathrm{L} \beta$-ACh (50 $\mathrm{nmol} / \mathrm{L})$ and $10 \mu \mathrm{L}$ caffeic acid $(10 \mu \mathrm{mol} / \mathrm{L})$ before precipitation with acetonitrile $(100 \mu \mathrm{L})$. The following procedures were the same as that for the brain tissue samples.

LC-MS/MS analysis

An Agilent UPLC 1290 systerm was interfaced to an Agilent QQQ-MS/MS (6460) equipped with an ESI ion source. The analysts were separated on a Agilent SB-C18 column $(100 \times 2.1 \mathrm{~mm}, 3.5 \mu \mathrm{m})$ with the column temperature set at $30^{\circ} \mathrm{C}$. The mobile phase was composed of solvent A $(0.1 \%$ formic acid and $10 \mathrm{mM}$ ammonium acetate in water) and solvent B (acetoniltrile). The mobile phase were eluted at 0.3 $\mathrm{mL} / \mathrm{min}$ following the gradient as follows: $10 \% \mathrm{~B}$ maintained for $3 \mathrm{~min}$, increased to $70 \%$ at $10 \mathrm{~min}$ and held for $3 \mathrm{~min}$, decreased to $10 \%$ at $13 \mathrm{~min}$ followed by $2 \mathrm{~min}$ for equilibration. The source operated in positive ion mode, and its main working parameters were set as follows: drying gas, nitrogen $\left(10 \mathrm{~L} / \mathrm{min}, 350^{\circ} \mathrm{C}\right)$; capillary voltage, $4 \mathrm{kV}$. Multiple reaction monitoring (MRM) measurements of the analytes were performed using individually optimized frangmentor energy and collision energy (see Table 1). Data acquisition was carried out by Agilent MassHunter workstation software (version B05.00). 


\section{Preparation of Total RNA and Quantitative Reverse Transcriptase PCR} (Real-Time PCR)

Total RNA was isolated from cortex and hippocampus, using the TRIzol® reagent (Invitrogen Co., Carlsbad, CA, USA) according to the manufacturer's instructions. The concentration and purity of total RNA were determined using NanoDrop 1000 (Thermo Scientific). The Rever TraAce qPCR RT kit (TOYOBO) was used for the reverse transcription of total RNA to cDNA. In real-time experiments, cDNA was analyzed in duplicate using $0.2 \mu \mathrm{mol} / \mathrm{L}$ specific primers (Table 2) and 1× LightCycler 480 SYBR green I Master (Roche Applied Science, Germany) in a total volume of $10 \mu 1$. $\beta$-actin was used as endogenous control. PCRs were carried out in a Light Cycle 480 (Roche Applied Science, Germany) using a thermal profile of $10 \mathrm{~min}$ at $95^{\circ} \mathrm{C}$ followed by 50 cycles of $15 \mathrm{~s}$ at $95^{\circ} \mathrm{C}, 30 \mathrm{~s}$ at $60^{\circ} \mathrm{C}$, a melting curve of $15 \mathrm{~s}$ at $95^{\circ} \mathrm{C}, 60 \mathrm{~s}$ at $1 \mathrm{~min}$, heating to $95^{\circ} \mathrm{C}$, and cooling for $30 \mathrm{~s}$ at $4^{\circ} \mathrm{C}$. The results were analyzed using the LightCycler 480 software (version 1.5, Roche Applied Science, Germany). Relative levels of mRNA were analyzed using the $\Delta \Delta \mathrm{Ct}$ method. The following primers were designed and synthesized by Sangon Biotech (Shanghai, China).

\section{Stastical Analysis}

Data were evaluated with SPSS 16.0 (SPSS, Inc., Chicago, IL, USA) and presented as the mean \pm SEM. In comparing the difference between the two groups, unpaired t test was used. $\mathrm{P}<0.05$ was considered statistically significant. 


\section{Results}

\section{Characterization of the Experimental Animals}

The STZ group animals present significantly higher values of blood glucose and a significant decrease in body weight gain when compared with control rats in the time points analyzed (Table 3), confirming the diabetic state of STZ animals began from 2 weeks after STZ treatment.

\section{Adversely Affected Cognitive Skills Appeared after 10 Weeks of STZ Treatment}

The STZ group animals and their age-matched control rats were assessed for cognitive function using MWM test for every two weeks, and the cognitive function was not affected until 10 weeks after STZ treatment (data not shown). In the 10-week STZ rats, there was a significant difference in latency time between two groups from the second day of testing (Fig. 1A). This result indicated that the STZ animals showed a decline ability to find the platform and learn its location in four-day training. In the probe trail of the MWM test, which assesses how well the animals have learned and consolidated the platform location during the four-days learning, diabetic rats showed impairment in memory. The percentage of time spent in the target quadrant (Fig. 1C) and the crossing times of the platform area (Fig. 1D) were significantly lower in 10-week diabetic group as compared with age-matched control group. In order to exclude difference in navigation speed, we also measured the mean swim speed (Fig. 1B) throughout the test. No significant difference was observed in the mean swim speed throughout the test, indicating that the previous parameters in the test were not due to a deficit in swimming speed. 


\section{Quantitative-Profiling of Diabetes-Related Neurotransmitter Alterations}

The levels of ACh, Glu and GABA were investigated in the serum, CSF, hippocampus and cortex every two weeks after STZ treatment to evaluate the alterations in neurotransmitter profiling during the process of disease progression (Fig. 2). In STZ rats, the ACh content was decreased in the serum, CSF, and brain regions as early as 6 weeks after STZ treatment (Fig. 2A-D). Diabetic also promoted a significant decrease in Glu level in the CSF and hippocampus at 10 weeks after STZ treatment, whereas no changes were observed in the serum and cortex until the end of the experiment (Fig. 2E-H). Further, the STZ treatment affected GABA level in different regions with different manners (Fig. 2I-L). Although GABA was significantly decreased in the serum of diabetic rats, its levels in the CSF, hippocampus and cortex were observed significantly $(\mathrm{p}<0.05)$ higher in STZ group compared to the age-matched control group. Moreover, the increase in GABA level was observed at 10 weeks in the CSF, 6 weeks in the hippocampus, and 4 weeks in the cortex.

\section{Effect of STZ Treatment on Neurotransmitter-Related Rate Limiting Enzyme}

It has been demonstrated that the synthetic and metabolism enzyme of neurotransmitter is neurochemically associated with the neurotransmitter level, and has a fundamental role in cognitive deficits observed in patients and in animal models of DM (Ghareeb and Hussen 2008). It has been reported that acetylcholinesterase (AChE) plays a central role in the regulation of ACh homeostasis(Li et al. 2015), and glutamate decarboxylase (GAD) catalyzes the biotransformation of Glu to GABA 
(Leke et al. 2014). In this study, we observed that the mRNA expressions of AChE, GAD1and GAD2 were all significantly up-regulated in the cortex and hippocampus of DE rats (Fig. 3).Our data indicated that the analysis of AChE and GAD expression in brain tissues might be used as markers of cholinergic, glutamatergic and

GABAergic activities respectively.

\section{Neuroinflammation in the STZ-induced DE Rats}

Emerging evidences have demonstrated that pro-inflammatory cytokines, such as IL-1 $\beta$ and TNF- $\alpha$, might lead to neuronal microenvironment alteration and seem to be a key factor in the development and progression of memory-related disorders(Vincenzi et al. 2014). In this study, brain cortex and hippocampus of DE animals contained approximately two times of the IL-1 $\beta$ compared to normal animals (Fig. 4A). Similarly, a significantly higher level of TNF- $\alpha$ was present in the cortex and hippocampus of DE rats $(\mathrm{p}<0.05)$ compared to control group (Fig. 4B).

\section{Discussion}

In human diabetes, chronic hyperglycemia is associated with a high incidence of progressive cognitive deficits (Kariharan et al. 2015). It has been reported that the neurotransmission alterations occurred earlier than structural and functional changes of cognitive deficits in most of degenerative encephalopathy (Shiihara et al. 2006). Therefore, this study was an attempt to catch sight of the neurotransmitter abnormalities in the progression of $\mathrm{DE}$, and to potentially provide novel noninvasive biomarkers for diagnosing the disease in its incipient stages. 
In the present study, we used a single injection of STZ to induce diabetes associated cognitive decline in rats. The STZ group presented a notable increase in glucose levels and a significant decrease in body weight as early as 2 weeks after STZ treatment, but the distinct impairment in learning and memory of diabetic animals were observed until 10 weeks after STZ treatment. This result demonstrated that the process of cognitive deficits is far hysteretic from diabetes development, and the diagnostic criteria of diabetes have little reference value to DE.

Investigators have established that neurotransmitter system dysfunction is one of the key neurochemical changes in most of the degenerative brain disease (Cochiolo et al. 2000). It has been reported that cholinergic and glutamatergic deficits are present at relatively early stages of $\mathrm{AD}$, while GABAergic systems appear to be affected later in the pathogenesis of AD (Martorana et al. 2009, Limon et al. 2012). But there is relatively little information concerning the neurotransmitter abnormalities in disease progression of DE. In this study, we found that ACh levels were decreased in CSF and brain tissues after six weeks of STZ administration. Notably, the same trend has also been observed in the serum of DE rats. The above data showed that the disturbance of ACh in peripheral blood is in parallel with the brain tissues, and the serum Ach might potentially be used as a novel noninvasive biomarker for DE diagnosis in the incipient stages.

Glu is the main excitatory neurotransmitter in the central nervous system, which participates in the maintenance of important physiological functions such as synaptic plasticity(Varga et al. 2014) and cognitive awareness(Abdallah et al. 2014). Previous 
study has reported that dysregulation of glutamate is implicated in receptor-mediated excitotoxicity in the progression of chronic neurodegenerative disorders (Gregory et al. 2013). In our study, we observed that Glu was decreased in hippocampus of DE rats, which is consistent with a recent report that showed a reduced level of Glu in hippocampal and cortical regions of diabetic rats(Datusalia and Sharma 2014).

Many studies support the concept of inhibition of memory formation by the GABAergic system. Drugs which modulate the GABAergic system at different stages like GABA synthesis, GABA binding site, or chloride channels significantly affect memory and cognitive ability(Cruz-Morales et al. 1993). Similarly, GABA antagonist and some GABA inhibiting steroids like pregenolone-sulfate show significant improvement in learning and memory (Flood et al. 1992). It was recently reported that there was a decrease in plasma GABA levels in patients with type 2 diabetes and impaired glucose tolerance when compared with control subjects(Menge et al. 2010). Another study shows that GABA release may represent a compensatory mechanism to prevent neuronal damage during hypoxia and ischemia(Ravindran et al. 1994). In our study, we found that the variation trend of GABA was completely opposite in central system (CSF, hippocampus and cortex) and peripheral system (plasma). The GABA levels were significantly decreased in the serum, but increased in the CSF, hippocampus and cortex at 10 weeks, 6 weeks and 4 weeks respectively in DE rats. Obviously, this in vivo data presents a possible supplement to specifically regulate GABA in the course of DE.

Several laboratories have noted that the abnormalities of neurotransmitters have 
been linked with the dysfunction of their related rate limiting enzymes. Early findings approved that brain tissues of $\mathrm{AD}$ patient revealed increased $\mathrm{AChE}$ expression and reduction ACh level in majority of cases (Giacobini 2003). The activity of AChE was also found evidently increased in the cerebral cortex of STZ induced diabetes rats (Kumar et al. 2013). In support of these findings, our results showed that the AChE activity was significantly increased in the cortex and hippocampus of 10-week STZ animals, which is likely to be responsible for the down-regulated ACh levels in DE rats. GAD expression can be used as a marker of overall Glutamatergic and GABAergic activity, and was found to be up-regulated in DE rats in the present study. As the central enzyme synthesizing GABA via decarboxylation of Glu, GAD is represented at two isoforms, GAD1 and GAD2. GAD1 catalyzes basic GABA formation for housekeeping neuronal functions, and GAD2 forms a complex with GABA vesicular transporter, favoring the coupling of GABA synthesis and transport into synaptic vesicles. Thus, it is possible that the increased GAD expression is the underlying cause for the alterations of Glu and GABA during the progression of DE in this study.

Activation of neuro-inflammation is very well reported and is known to involve in peripheral and CNS complications of diabetes (Kumar and Sharma 2010). Evidences have proved that prolonged uncontrolled hyperglycemia may active the NF-кB signaling pathway, and furthermore, affect the pathophysiology of diabetes

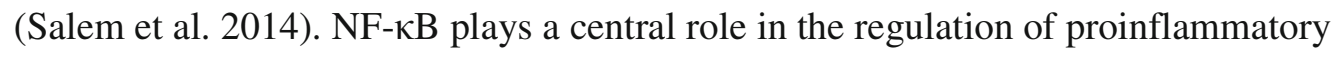
and inflammatory mediators such as TNF- $\alpha$ and IL- $1 \beta$, which leads to alteration in 
neuronal microenvironment and seems to be a key factor in the development and progression of memory-related disorders (Negi et al. 2011, Yirmiya and Goshen 2011). It has been reported that hypomethylation of IL-1 $\beta$ is strongly associated with chronological age and with elevated IL-1 $\beta$ transcription in humans. (Cho et al. 2015). Another study also demonstrated that isoflurane-induced cognitive deficits may stem from upregulation of hippocampal IL-1 $\beta$, partially via activation of the

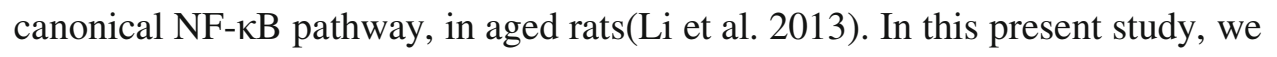
observed a significant increase in the expression of TNF- $\alpha$ and IL-1 $\beta$ in the brain tissues of DE rats, which also support the negative correlation of the elevated inflammatory markers with reduced cognitive function in the experimental diabetes rats. Although we have demonstrated that neuroinflammation contributes to diabetes-induced cognitive deficits, additional studies are needed to determine the underlying mechanism of this inflammatory process.

In conclusion, the data presented here strongly supports that cognitive deficits induced by diabetes have a close relationship with neurotransmitter homeostasis and metabolism. Different neurotransmitter may be differentially modulated, with differences in magnitude, time course, and effect. Therefore, we believe that the well recognition of neurotransmitter regulation in DE is not only meaningful for better therapeutic regiment design and early diagnosis, but may also shed a light on understanding the pathological process of the disease. 


\section{Acknowledgements}

This work was supported by the Natural Science Foundation of China (No. 81403001), the Natural Science Foundation of the Jiangsu Higher Education Institutions of China (No. 13KJB350007), and A Project Funded by the Priority Academic Program Development of Jiangsu Higher Education Institutions (PAPD). The authors have declared no conflict of interest.

\section{References}

Abdallah, C. G., Niciu, M. J., Fenton, L. R., Fasula, M. K., Jiang, L., Black, A., et al. 2014. Decreased occipital cortical glutamate levels in response to successful cognitive-behavioral therapy and pharmacotherapy for major depressive disorder. Psychother. Psychosom. 83(5): 298-307. DOI: 10.1159/000361078

Alberdi, E., Sanchez-Gomez, M. V., Cavaliere, F., Perez-Samartin, A., Zugaza, J. L., Trullas, R., et al. 2010. Amyloid beta oligomers induce Ca2+ dysregulation and neuronal death through activation of ionotropic glutamate receptors. Cell Calcium. 47(3): 264-272. DOI: 10.1016/j.ceca.2009.12.010

Antila, K., Lotjonen, J., Thurfjell, L., Laine, J., Massimini, M., Rueckert, D., et al. 2013. The PredictAD project: development of novel biomarkers and analysis software for early diagnosis of the Alzheimer's disease. Interface Focus. 3(2): 20120072. DOI: 10.1098/rsfs.2012.0072

Biessels, G. J., Deary, I. J. and Ryan, C. M. 2008. Cognition and diabetes: a lifespan perspective. Lancet Neurol. 7(2): 184-190 DOI: 10.1016/S1474-4422(08)70021-8 
Cho,S.H. , Chen, J.A., Sayed, F., Ward, M.E., Gao, F., Nguyen, T.A., et al. 2015. SIRT1 deficiency in microglia contributes to cognitive decline in aging and neurodegeneration via epigenetic regulation of IL-1beta. J Neurosci. 35(2): 807-818. doi: 10.1523/JNEUROSCI.2939-14.2015.

Cochiolo, J. A., Ehsanian, R. and Bruck, D. K. 2000. Acute ultrastructural effects of MPTP on the nigrostriatal pathway of the C57BL/6 adult mouse: evidence of compensatory plasticity in nigrostriatal neurons. J. Neurosci. Res. 59(1): 126-135. PMID: 10658193

Cruz-Morales, S. E., Quirarte, G. L., Diaz del Guante, M. A. and Prado-Alcala, R. A. 1993. Effects of GABA antagonists on inhibitory avoidance. Life Sci. 53(16): 1325-1330. PMID: 8412493

Datusalia, A. K. and Sharma, S. S. 2014. Amelioration of diabetes-induced cognitive deficits by GSK-3beta inhibition is attributed to modulation of neurotransmitters and neuroinflammation. Mol. Neurobiol. 50(2): 390-405. DOI: $10.1007 / \mathrm{s} 12035-014-8632-\mathrm{X}$

Flood, J. F., Morley, J. E. and Roberts, E. 1992. Memory-enhancing effects in male mice of pregnenolone and steroids metabolically derived from it. Proc. Natl. Acad. Sci. U S A. 89(5): 1567-1571. PMID: 1531874

Frigerio, F., Casimir, M., Carobbio, S. and Maechler, P. 2008. Tissue specificity of mitochondrial glutamate pathways and the control of metabolic homeostasis. Biochim. Biophys. Acta. 1777(7-8): 965-972. DOI: 10.1016/j.bbabio.2008.04.031

Ghareeb, D. A. and Hussen, H. M. 2008. Vanadium improves brain 
acetylcholinesterase activity on early stage alloxan-diabetic rats. Neurosci. Lett. 436(1): 44-47. DOI: 10.1016/j.neulet.2008.02.073

Giacobini, E. 2003. Cholinesterases: new roles in brain function and in Alzheimer's disease. Neurochem. Res. 28(3-4): 515-522. PMID: 12675140

Gispen, W. H. and Biessels, G.J. 2000. Cognition and synaptic plasticity in diabetes mellitus. Trends Neurosci. 23(11): 542-549. PMID: 11074263

Gregory, K. J., Noetzel, M. J. and Niswender, C. M. 2013. Pharmacology of metabotropic glutamate receptor allosteric modulators: structural basis and therapeutic potential for CNS disorders. Prog. Mol. Biol. Transl. Sci. 115: 61-121. DOI: 10.1016/B978-0-12-394587-7.00002-6

Jangra, A., Datusalia, A.K., Khandwe, S. and Sharma, S.S. 2013. Amelioration of diabetes-induced neurobehavioral and neurochemical changes by melatonin and nicotinamide: implication of oxidative stress-PARP pathway. Pharmacol Biochem Behav. 114-115: 43-51. doi: 10.1016/j.pbb.2013.10.021.

Kariharan, T., Nanayakkara, G., Parameshwaran, K., Bagasrawala, I., Ahuja, M., Abdel-Rahman, E., et al. 2015. Central activation of PPAR-gamma ameliorates diabetes induced cognitive dysfunction and improves BDNF expression. Neurobiol. Aging. 36(3): 1451-1461. DOI: 10.1016/j.neurobiolaging.2014.09.028

Kodl, C. T. and Seaquist, E. R. 2008. Cognitive dysfunction and diabetes mellitus. Endocr. Rev. 29(4): 494-511. DOI: 10.1210/er.2007-0034

Kumar, A., Chaudhary, T. and Mishra, J. 2013. Minocycline modulates neuroprotective effect of hesperidin against quinolinic acid induced Huntington's 
disease like symptoms in rats: behavioral, biochemical, cellular and histological evidences. Eur. J. Pharmacol. 720(1-3): 16-28. DOI: 10.1016/j.ejphar.2013.10.057

Kumar, A. and Sharma, S. S. 2010. NF-kappaB inhibitory action of resveratrol: a probable mechanism of neuroprotection in experimental diabetic neuropathy. Biochem. Biophys. Res. Commun. 394(2): 360-365. DOI:

10.1016/j.bbrc.2010.03.014

Leke, R., Silveira, T. R., Escobar, T. D. and Schousboe, A. 2014. Expression of Glutamate Decarboxylase (GAD) mRNA in the brain of bile duct ligated rats serving as a model of hepatic encephalopathy. Neurochem. Res. 39(3): 605-611. DOI: $10.1007 / \mathrm{s} 11064-013-1116-y$

Levenga, J., Krishnamurthy, P., Rajamohamedsait, H., Wong, H., Franke, T. F., Cain, P., et al. 2013. Tau pathology induces loss of GABAergic interneurons leading to altered synaptic plasticity and behavioral impairments. Acta. Neuropathol.

Commun. 1(1): 34. DOI: 10.1186/2051-5960-1-34

Li, Y., Jiang, L. J., Yuan, L., Cao, T. T., Li, X., Dong, L., et al. 2015. Inhibition of acetylcholinesterase (AChE): A Potential Therapeutic Target toTreat Alzheimer's Disease. Chem. Biol. Drug. Des. DOI: 10.1111/cbdd.12550

Li, Z.Q., Rong, X.Y., Liu, Y.J., Ni, C., Tian, X.S., Mo, N., et al.2013. Activation of the canonical nuclear factor-kappaB pathway is involved in isoflurane-induced hippocampal interleukin-1 beta elevation and the resultant cognitive deficits in aged rats. Biochem Biophys Res Commun 438(4): 628-634. doi: 10.1016/j.bbrc.2013.08.003. 
Liapi, C., Kyriakaki, A., Zarros, A., Galanopoulou, P., Al-Humadi, H., Dontas, I., et al. 2010. Choline-deprivation alters crucial brain enzyme activities in a rat model of diabetic encephalopathy.Metab Brain Dis 25(3): 269-276. doi:

10.1007/s11011-010-9205-y.

Limon, A., Reyes-Ruiz, J. M. and Miledi, R. 2012. Loss of functional GABA(A) receptors in the Alzheimer diseased brain. Proc. Natl. Acad. Sci. U S A. 109(25): 10071-10076. DOI: 10.1073/pnas.1204606109

Liu, Y. W., Zhu, X., Li, W., Lu, Q., Wang, J. Y., Wei, Y. Q. , et al. 2012. Ginsenoside Re attenuates diabetes-associated cognitive deficits in rats. Pharmacol. Biochem. Behav. 101(1): 93-98. DOI: 10.1016/j.pbb.2011.12.003

Liu, Y. W., Zhu, X., Yang, Q. Q., Lu, Q., Wang, J. Y., Li, H. P., et al. 2013. Suppression of methylglyoxal hyperactivity by mangiferin can prevent diabetes-associated cognitive decline in rats. Psychopharmacology (Berl). 228(4): 585-594. DOI: 10.1007/s00213-013-3061-5

Martorana, A., Mori, F., Esposito, Z., Kusayanagi, H., Monteleone, F., Codeca, C., et al. 2009. Dopamine modulates cholinergic cortical excitability in Alzheimer's disease patients. Neuropsychopharmacology. 34(10): 2323-2328. DOI: 10.1038/npp.2009.60

McCrimmon, R. J. e. a. 2012. Diabetes and cognitive dysfunction. Lancet. 379(9833): 2291-2299. DOI: 10.1016/S0140-6736(12)60360-2

Menge, B. A., Schrader, H., Ritter, P. R., Ellrichmann, M., Uhl, W., Schmidt, W. E. , et al. 2010. Selective amino acid deficiency in patients with impaired glucose 
tolerance and type 2 diabetes. Regul. Pept. 160(1-3): 75-80. DOI:

10.1016/j.regpep.2009.08.001

Negi, G., Kumar, A. and Sharma, S. S. 2011. Melatonin modulates neuroinflammation and oxidative stress in experimental diabetic neuropathy: effects on NF-kappaB and Nrf2 cascades. J. Pineal. Res. 50(2): 124-131. DOI:

10.1111/j.1600-079X.2010.00821.X

Perry, E., Walker, M., Grace, J. and Perry, R. 1999. Acetylcholine in mind: a neurotransmitter correlate of consciousness? Trends. Neurosci. 22(6): 273-280. PMID: 10354606

Prakash, A., Kalra, J., Mani, V., Ramasamy, K. and Majeed, A. B. 2015. Pharmacological approaches for Alzheimer's disease: neurotransmitter as drug targets. Expert. Rev. Neurother. 15(1): 53-71. DOI:

$10.1586 / 14737175.2015 .988709$

Ramamoorthi, K. and Lin, Y. 2011. The contribution of GABAergic dysfunction to neurodevelopmental disorders. Trends Mol Med 17(8): 452-462. doi: 10.1016/j.molmed.2011.03.003

Ravindran, J., Shuaib, A., Ijaz, S., Galazka, P., Waqar, T., Ishaqzay, R., et al. 1994. High extracellular GABA levels in hippocampus--as a mechanism of neuronal protection in cerebral ischemia in adrenalectomized gerbils. Neurosci. Lett. 176(2): 209-211. PMID: 7830949

Reisi, P., Alaei, H., Babri, S., Sharifi, M.R., Mohaddes, G. and Soleimannejad, E. 2009. Determination of the extracellular basal levels of glutamate and GABA at 
dentate gyrus of streptozotocin-induced diabetic rats. Pathophysiology 16(1): 63-66. doi: 10.1016/j.pathophys.2009.03.003.

Salem, H.H., Trojanowski, B., Fiedler, K., Maier, H.J., Schirmbeck, R., Wagner, M., et al. 2014. Long-term IKK2/NF-kappaB signaling in pancreatic beta-cells induces immune-mediated diabetes. Diabetes 63(3): 960-975. doi: 10.2337/db13-1037

Shiihara, T., Kato, M., Ichiyama, T., Takahashi, Y., Tanuma, N., Miyata, R. , et al. 2006. Acute encephalopathy with refractory status epilepticus: bilateral mesial temporal and claustral lesions, associated with a peripheral marker of oxidative DNA damage. J. Neurol. Sci. 250(1-2): 159-161. DOI: 10.1016/j.jns.2006.07.002

Singhal, K. and Sandhir, R. 2015. L-type calcium channel blocker ameliorates diabetic encephalopathy by modulating dysregulated calcium homeostasis. J Neurosci. Res. 93(2): 296-308. DOI: 10.1002/jnr.23478

Varga, E., Juhasz, G., Bozso, Z., Penke, B., Fulop, L. and Szegedi, V. 2014. Amyloid-beta1-42 Disrupts Synaptic Plasticity by Altering Glutamate Recycling at the Synapse. J. Alzheimers. Dis. DOI: 10.3233/JAD-142367

Vincenzi, B., Stock, S., Borba, C. P., Cleary, S. M., Oppenheim, C. E., Petruzzi, L. J., et al. 2014. A randomized placebo-controlled pilot study of pravastatin as an adjunctive therapy in schizophrenia patients: effect on inflammation, psychopathology, cognition and lipid metabolism. Schizophr. Res. 159(2-3): 395-403. DOI: 10.1016/j.schres.2014.08.021

Wan, Y., Wang, Q. and Prud'homme, G. J. 2015. GABAergic system in the endocrine pancreas: a new target for diabetes treatment. Diabetes. Metab. Syndr. Obes. 8: 


\section{9-87. DOI: 10.2147/DMSO.S50642}

Welsh, B. and Wecker, L. 1991. Effects of streptozotocin-induced diabetes on acetylcholine metabolism in rat brain. Neurochem Res 16(4): 453-460. PMID: 1833657

Yirmiya, R. and Goshen, I. 2011. Immune modulation of learning, memory, neural plasticity and neurogenesis. Brain. Behav. Immun. 25(2): 181-213. DOI:

10.1016/j.bbi.2010.10.015

Zheng, X., Kang, A., Dai, C., Liang, Y., Xie, T., Xie, L., et al. 2012. Quantitative analysis of neurochemical panel in rat brain and plasma by liquid chromatography-tandem mass spectrometry. Anal. Chem. 84(22): 10044-10051. DOI: $10.1021 / \mathrm{ac} 3025202$ 


\section{Tables}

Table 1 The analytic parameters of neurotransmitters by LC-MS/MS

\begin{tabular}{cccccc}
\hline Analyte & Precursor & Product & Fragmentor & Dwell & Collision \\
& ion(m/z) & ion(m/z) & energy(v) & time(ms) & energy(v) \\
\hline ACh & 146 & 87 & 85 & 200 & 15 \\
Glu & 252 & 105 & 80 & 200 & 10 \\
GABA & 208 & 105 & 75 & 200 & 10 \\
$\beta$-ACh & 160.1 & 101 & 75 & 200 & 10 \\
caffeic acid & 406 & 105 & 110 & 100 & 20 \\
\hline
\end{tabular}


Table 2 Primer sequences used for gene expression analysis

\begin{tabular}{|c|c|c|}
\hline \multirow{2}{*}{ Gene } & \multicolumn{2}{|c|}{ Prime sequences (5'-3') } \\
\hline & Forward & Reverse \\
\hline $\mathrm{AChE}$ & GGCCTACTTCTCCCACACCT & ATACCAACACGGTTCCCTCA \\
\hline Gad1 & AAGATGATGGGTGTGCTGCT & ACTGCTTGTCTGGCTGGAAG \\
\hline Gad2 & TCGGAAACACAAGTGGAAGC & GACCAGGAGAGCCGAACATT \\
\hline TNF- $\alpha$ & GCGTGTTCATCCGTTCTCTAC & CGTCTCGTGTGTTTCTGAGC \\
\hline $\mathrm{IL}-1 \beta$ & GTCAАCTCCATCTGCCCTTC & TGTGGGTGGTATCCTCTGTG \\
\hline$\beta$-actin & CCCATCTATGAGGGTTACGC & TTTAATGTCACGCACGATTTC \\
\hline
\end{tabular}


Table 3 Body weight and blood glucose level of STZ group rats and age-matched

\begin{tabular}{|c|c|c|c|c|}
\hline \multirow[b]{2}{*}{ group } & \multicolumn{2}{|c|}{ Body weight（g） } & \multicolumn{2}{|c|}{ Blood glucose (mmol/L) } \\
\hline & control & STZ & control & STZ \\
\hline 2 weeks & $270.67 \pm 6.29$ & $181.17 \pm 8.42^{* *}$ & $4.48 \pm 0.36$ & $19.27 \pm 1.38^{* *}$ \\
\hline 4 weeks & $259.17 \pm 10.06$ & $195.00 \pm 7.94^{*}$ & $5.05 \pm 0.42$ & $19.25 \pm 0.77^{* *}$ \\
\hline 6 weeks & $300.17 \pm 4.30$ & $171.17 \pm 6.71^{* *}$ & $5.84 \pm 0.23$ & $23.19 \pm 1.65^{* *}$ \\
\hline 8 weeks & $299.00 \pm 13.02$ & $160.86 \pm 12.62^{* *}$ & $5.07 \pm 0.13$ & $21.05 \pm 0.92^{* *}$ \\
\hline 10 weeks & $397.83 \pm 6.37$ & $162.00 \pm 6.92^{* *}$ & $5.19 \pm 0.32$ & $22.82 \pm 1.22^{* *}$ \\
\hline
\end{tabular}

Data are means \pm S.E.M. of 6 to 8 animals from each condition studied.

Statistical significance: $* * \mathrm{P}<0.01, * \mathrm{P}<0.05$ when compared with control rats. 


\section{Figure captions}

Fig. 1 Effects of STZ treatment on the latency time (A), swimming speed (B), percentage of time spent in target quadrant (C) and crossing times of the platform area (D) in MWM test of STZ group (treated with a single intraperitoneal injection of 60 $\mathrm{mg} / \mathrm{kg} \mathrm{STZ}$ ) and control group (received $0.1 \mathrm{M}$ citrate buffer at $\mathrm{pH} 4.5$ only). Data are presented as means \pm S.E.M. of 6 to 8 animals in each group. $* \mathrm{P}<0.05,{ }^{*} \mathrm{P}<0.01$, vs. age-matched control group.

Fig. 2 Quantitative-profiling of the neurotransmitter levels in experimental rats. STZ group was treated with a single intraperitoneal injection of STZ $(60 \mathrm{mg} / \mathrm{kg}$, dissolved in $0.1 \mathrm{M}$ citrate buffer at $\mathrm{pH} 4.5$ ). Age-matched control rats received vehicle only. The serum (A,E,I), CSF (B,F,J), hippocampus $(\mathrm{C}, \mathrm{G}, \mathrm{K})$ and cortex $(\mathrm{D}, \mathrm{H}, \mathrm{L})$ were collected at designated times $(2,4,6,8$ and 10 weeks) for ACh, Glu and GABA determination. Data are presented as means \pm S.E.M. of 6 to 8 animals in each group.

Statistical significance: $* \mathrm{P}<0.05, * * \mathrm{P}<0.01$, vs. age-matched control group.

Fig.3 The mRNA expression of AChE(A), GAD1(B) and GAD2(C) in the cortex and hippocampus of STZ group (treated with a single intraperitoneal injection of 60 $\mathrm{mg} / \mathrm{kg} \mathrm{STZ}$ ) and control group (received $0.1 \mathrm{M}$ citrate buffer at $\mathrm{pH} 4.5$ only). Data are presented as means \pm S.E.M. of 6 to 8 animals in each group. $\quad * \mathrm{P}<0.05, * * \mathrm{P}<0.01$, vs. age-matched control group.

Fig. 4 The mRNA expression of IL-1 $\beta$ (A) and TNF- $\alpha$ (B) in the cortex and hippocampus of experimental rats. Data are presented as means \pm S.E.M. of 6 to 8 animals in each group. $\quad * \mathrm{P}<0.05, * * \mathrm{P}<0.01$, vs. age-matched control group. 

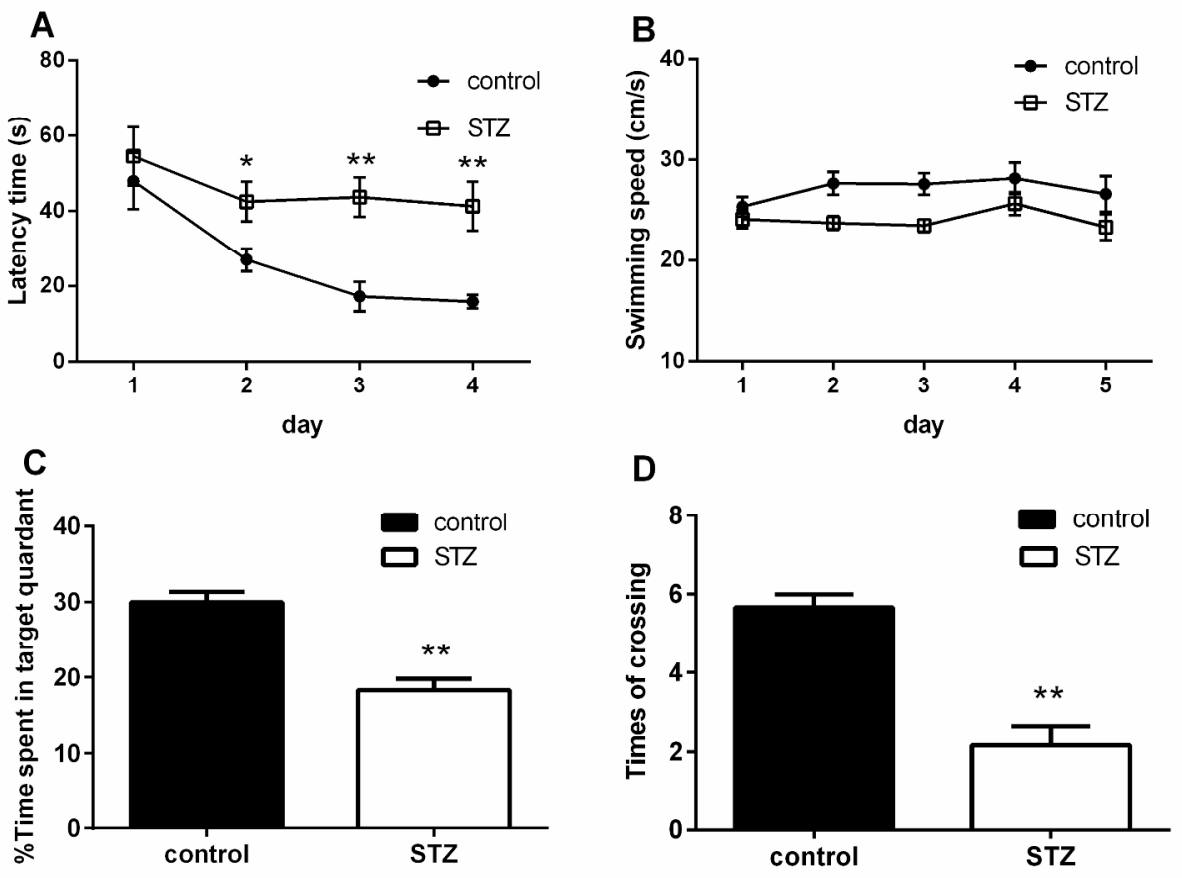

Effects of STZ treatment on the latency time (A), swimming speed (B), percentage of time spent in target quadrant (C) and crossing times of the platform area (D) in MWM test of STZ group (treated with a single intraperitoneal injection of $60 \mathrm{mg} / \mathrm{kg} \mathrm{STZ}$ ) and control group (received $0.1 \mathrm{M}$ citrate buffer at pH $4.5 \mathrm{only}$ ).

Data are presented as means \pm S.E.M. of 6 to 8 animals in each group. $* P<0.05, * * P<0.01$, vs. agematched control group.

$261 \times 185 \mathrm{~mm}(300 \times 300 \mathrm{DPI})$ 

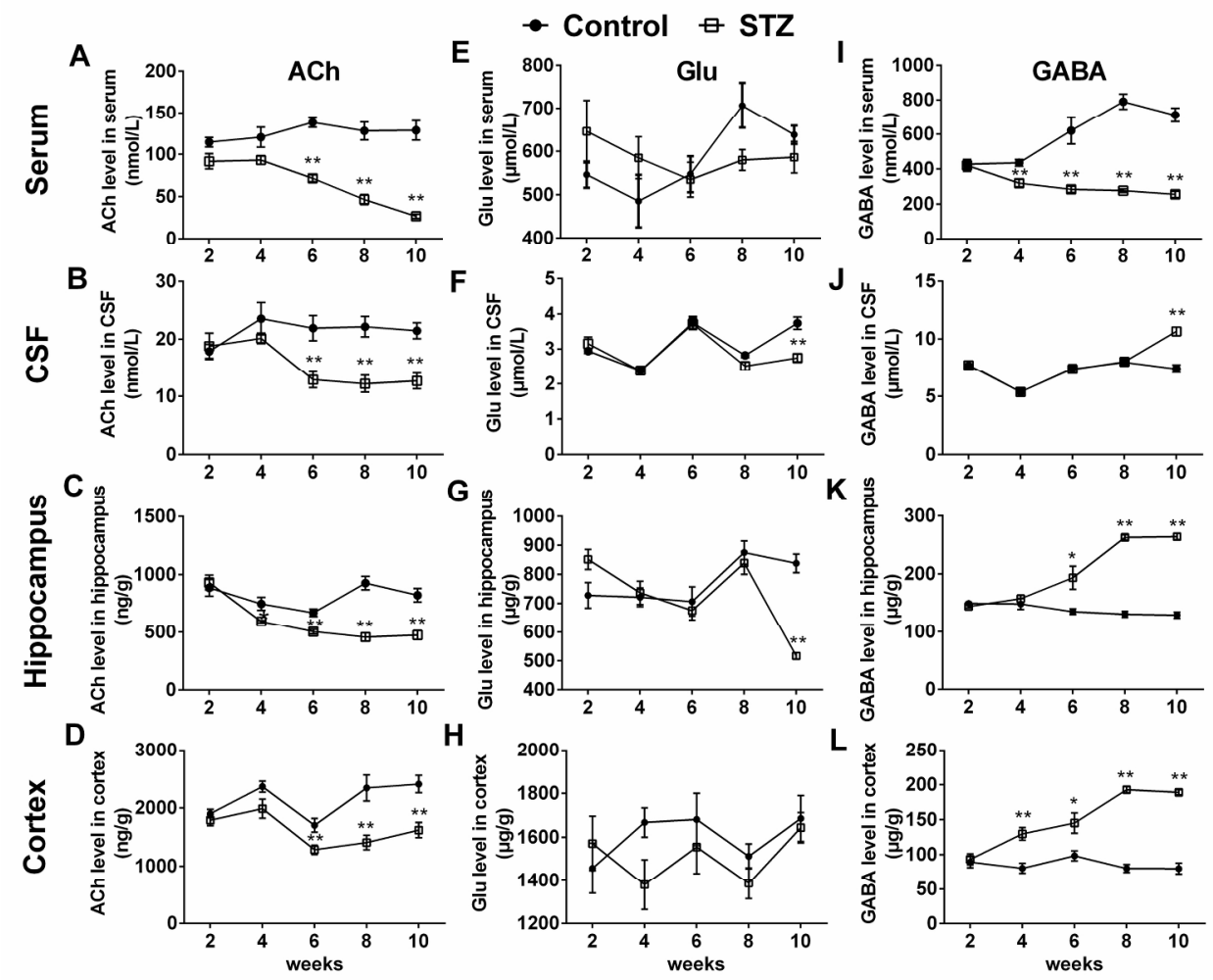

$214 \times 170 \mathrm{~mm}(300 \times 300$ DPI $)$ 

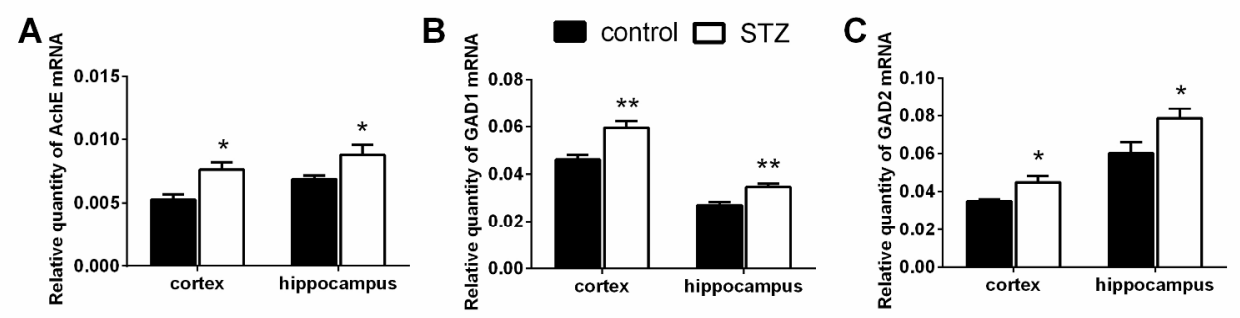

The mRNA expression of AChE(A), GAD1(B) and GAD2(C) in the cortex and hippocampus of STZ group (treated with a single intraperitoneal injection of $60 \mathrm{mg} / \mathrm{kg} \mathrm{STZ}$ ) and control group (received $0.1 \mathrm{M}$ citrate buffer at pH 4.5 only). Data are presented as means \pm S.E.M. of 6 to 8 animals in each group. ${ }^{*} \mathrm{P}<0.05$, $* * \mathrm{P}<0.01$, vs. age-matched control group.

$242 \times 65 \mathrm{~mm}(300 \times 300 \mathrm{DPI})$ 

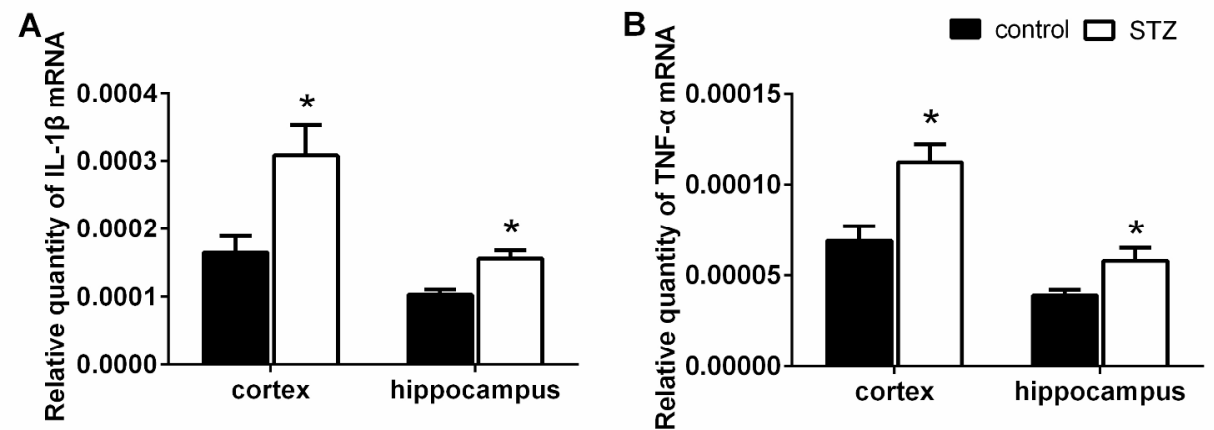

The mRNA expression of IL-1 $\beta$ (A) and TNF-a (B) in the cortex and hippocampus of experimental rats. Data are presented as means \pm S.E.M. of 6 to 8 animals in each group. $* P<0.05, * * P<0.01$, vs. age-matched control group.

$252 \times 93 \mathrm{~mm}(300 \times 300 \mathrm{DPI})$ 